FTUAM-16-30

IFT-UAM/CSIC-16-078

\title{
Prospects for Detecting Galactic Sources of Cosmic Neutrinos with IceCube: An Update
}

\author{
Francis Halzen $^{\mathrm{a}}$, Ali Kheirandish ${ }^{\mathrm{a}}$, Viviana Niro ${ }^{\mathrm{b}}$ \\ ${ }^{a}$ Wisconsin IceCube Particle Astrophysics Center and Department of Physics, University of Wisconsin, Madison, WI 53706, USA \\ ${ }^{b}$ Departamento de Física Teórica, Universidad Autónoma de Madrid, and Instituto de Física Teórica UAM/CSIC, Calle Nicolás \\ Cabrera 13-15, Cantoblanco, E-28049 Madrid, Spain
}

\begin{abstract}
Air-Cherenkov telescopes have mapped the Galactic plane at $\mathrm{TeV}$ energies. Here we evaluate the prospects for detecting the neutrino emission from sources in the Galactic plane assuming that the highest energy photons originate from the decay of pions, which yields a straightforward prediction for the neutrino flux from the decay of the associated production of charged pions. Four promising sources are identified based on having a large flux and a flat spectrum. We subsequently evaluate the probability of their identification above the atmospheric neutrino background in IceCube data as a function of time. We show that observing them over the twenty-year lifetime of the instrumentation is likely, and that some should be observable at the $3 \sigma$ level with six years of data. In the absence of positive results, we derive constraints on the spectral index and cut-off energy of the sources, assuming a hadronic acceleration mechanism.
\end{abstract}

Keywords: High-energy neutrinos; Neutrino astronomy; High-energy cosmic-ray physics and astrophysics

\section{Introduction}

The rationale for building kilometer-scale neutrino detectors is that their sensitivity is sufficient to reveal generic cosmic-ray sources with an energy density in neutrinos comparable to their energy density in cosmic rays and pionic $\mathrm{TeV}$ gamma rays. Interestingly, this condition should be satisfied by the sources of Galactic cosmic rays.

The energy density of the cosmic rays in our Galaxy is $\rho_{E} \sim 10^{-12} \mathrm{erg} \mathrm{cm}^{-3}$. Galactic cosmic rays do not exist forever; they diffuse within microgauss fields and remain trapped for an average containment time of $3 \times 10^{6}$ years. The power needed to maintain a steady energy density requires accelerators delivering $10^{41} \mathrm{erg} / \mathrm{s}$. This happens to be $10 \%$ of the power produced by supernovae releasing $10^{51}$ erg every 30 years $\left(10^{51} \mathrm{erg}\right.$ correspond to $1 \%$ of the binding energy of a neutron star after $99 \%$ is initially lost to neutrinos). This coincidence is the basis for the idea that shocks produced by supernovae exploding into the interstellar medium are the accelerators of Galactic cosmic rays.

A generic supernova remnant releasing an energy of $W \sim 10^{50} \mathrm{erg}$ into the acceleration of cosmic rays will inevitably generate $\mathrm{TeV}$ gamma rays by interacting with the hydrogen in the Galactic disk. Here every neutral pion in the dump produces two photons with half its energy. The emissivity, the number of photons produced per unit volume and time, is simply proportional to the density of cosmic rays and to the target density of hydrogen atoms. Integrating over the volume of the remnant we obtain an estimate of the $\mathrm{TeV}$ gamma ray flux from a

Email addresses: francis.halzen@icecube.wisc.edu (Francis Halzen), ali.kheirandish@icecube.wisc.edu (Ali Kheirandish), viviana.niro@uam.es (Viviana Niro) 
supernova remnant at a distance $d$ :

$$
\begin{aligned}
& \int_{E>1 \mathrm{TeV}} \frac{d N_{\gamma}}{d E_{\gamma}} d E_{\gamma}=\frac{L_{\gamma}(>1 \mathrm{TeV})}{4 \pi d^{2}} \\
& \simeq 10^{-12}-10^{-11}\left(\frac{\text { photons }}{\mathrm{cm}^{2} \mathrm{~s}}\right)\left(\frac{W}{10^{50} \mathrm{erg}}\right)\left(\frac{n}{1 \mathrm{~cm}^{-3}}\right)\left(\frac{d}{1 \mathrm{kpc}}\right)^{-2} ;
\end{aligned}
$$

for a detailed discussion see reference $[1,2]$.

The position of the knee in the cosmic ray spectrum indicates that some sources accelerate cosmic rays to energies of several PeV. These PeVatrons therefore produce pionic gamma rays whose spectrum should extend to several hundred TeV. Like for gamma rays, the search for Galactic neutrino sources concentrates on the search for PeVatrons, supernova remnants with the required energetics to produce cosmic rays, at least up to the knee in the spectrum. Some may have been revealed by the highest energy all-sky survey in $\sim 10 \mathrm{TeV}$ gamma rays using the Milagro detector [3]. A subset of nearby sources, located within nearby star-forming regions in Cygnus and in the vicinity of Galactic latitude $l=40$ degrees, are identified; some cannot be readily associated with known supernova remnants or with nonthermal sources observed at other wavelengths. Subsequently, directional air Cherenkov telescopes were pointed at three of the sources, revealing them as PeVatron candidates with an approximate $E^{-2}$ energy spectrum that extends to tens of $\mathrm{TeV}$ without evidence for a cutoff as we will explain further on.

Some Milagro sources may actually be molecular clouds illuminated by the cosmic-ray beam accelerated in young remnants located within $\sim 100 \mathrm{pc}$. Indeed, one expects that multi-PeV cosmic rays are accelerated only over a short time period when the shock velocity is high, i.e., when the remnant transitions from free expansion to the beginning of the Sedov phase. The high-energy particles can produce photons and neutrinos over much longer periods when they diffuse through the interstellar medium to interact with nearby molecular clouds [4]. An association of molecular clouds and supernova remnants is expected, of course, in star-forming regions. In this case, any confusion with synchrotron and inverse compton photons is unlikely.

Ground-based and satellite-borne instruments with improved sensitivity are able to conclusively pinpoint supernova remnants as the sources of cosmic-ray acceleration by identifying accompanying gamma rays of pion origin. The Fermi Large Area Telescope has detected pion-decay feature in the gamma-ray spectra of two supernova remnants, IC 443 and W44 [5]. In contrast, GeV gamma ray data from Fermi LAT have challenged the hadronic interpretation of the GeV-TeV radiation from one of the best-studied candidates, RX J1713-3946 [6]. The most promising PeVatron candidate to date is, instead, the center of the Galaxy, as reported by the HESS Collaboration, see Ref. [7]. Detecting the accompanying neutrinos from supernova remnants or the Galactic Centre would provide incontrovertible evidence for cosmic-ray acceleration.

Particle physics dictates the relation between pionic gamma rays and neutrinos and basically predicts the production of a $\nu_{\mu}+\bar{\nu}_{\mu}$ pair for every two gamma rays seen by Milagro. The results simply follows from the fact that for every neutral pion producing two photons, two charged pions are produced through the decay chain $\pi^{+} \rightarrow \nu_{\mu}+\mu^{+} \rightarrow \nu_{\mu}+\left(e^{+}+\nu_{e}+\bar{\nu}_{\mu}\right)$. A detailed calculation reveals a ratio of neutrinos to gamma rays that is reduced to 0.4 because of the production of photons by $\eta$ mesons. A variety of estimates $[1,8,9]$, which we update in this paper, predict that the sources can be detected aver several years. Two Milagro that are positioned close to the horizon for a detector at the South Pole, MGRO J1852+01 and MGRO J1908+06, are especially promising because photons with energy in excess of $40 \mathrm{TeV}$ can reach the detector without significant absorption in the Earth. This is not the case for the sources in the Cygnus region.

The IceCube experiment has discovered a flux of high-energy neutrinos from extragalactic sources with an energy density similar to that observed for gamma rays [10]. The observation underscores the important role of sources accelerating protons that produce similar energy in photons and neutrinos, which are the decay products of neutral and charged pions, respectively.

The present data cannot exclude a subdominant flux of Galactic origin in the IceCube data [11-14]. Unidentified sources [15], Fermi bubbles [11, 16, 17], and Sagittarius $A^{*}[18]$ have been reviewed as potential Galactic sources. However, the general conclusion is that these sources can account for a fraction of the events detected. Specifically, the possibility that the hot spot close to the Galactic Center (GC) is produced by a single point source with a flux normalization of $6 \times 10^{-8} \mathrm{GeV} \mathrm{cm}^{-2} \mathrm{~s}^{-1}$ has been excluded [19,20]. 
In a map of the northern Galactic plane obtained with Milagro data, six promising neutrino sources were identified in Refs. [1, 8]. The IceCube Collaboration has carried out extensive searches for point and extended sources in Ref. [21], reporting evidence with a significance of $2.5 \sigma$, when the six Milagro sources are considered together [21].

In the previous study, Ref. [20], the authors revisited the prospects for observing the three confirmed Milagro sources and re-evaluated the probability and constraints in light of the low-energy cut-off reported by the Milagro collaboration $[22,23]$. The authors concluded that more than 10 years of running IceCube is necessary to yield a discovery at the level of $3 \sigma$. In the case of the source MGRO J1908+06, evidence at $3 \sigma$ could be obtained in seven years assuming values of the spectral index and the cut-off energy that are in good agreement with the best fit reported in [22].

In this paper, we will update the theoretical predictions using the observation and flux measurements reported by HAWC, ARGO-YBJ, and air Cherenkov telescopes (ACT) VERITAS and HESS. Most importantly, with a detector superior to Milagro, the HAWC experiment has confirmed only four of the six sources [24, 25]: MGRO J1908+06, MGRO J1852+01, MGRO J2031+41, and MGRO J2019+37. For these, we will construct a gamma ray spectrum based on all information available and evaluate the neutrino flux. Subsequently, we will compute the number of signal and background events as well as the p-value for observing the sources as a function of time. Finally, we will determine exclusion limits on a flux of hadronic origin in the absence of an observation. Our main results can be summarized as follows:

- MGRO J1908+06: Although historically classified as a pulsar wind nebula (PWN) and currently as an unidentified source, its large size and hard spectrum in $\mathrm{TeV}$ photons suggest that it may be a supernova remnant (SNR). SNRs are suspected to be the sources of the highest energy cosmic rays in the Galaxy. We re-evaluate the probability of observing the source using the flux reported by HESS and anticipate a $3 \sigma$ observation in about 10 years of IceCube data. However, the answer depends on the actual threshold of the specific analysis. By increasing the energy threshold, IceCube has the potential to observe MGRO J1908+06 at the some statistical level with only six years of data. A lack of observation in 15 years of IceCube data will indicate that MGRO J1908+06 is not a cosmic-ray accelerator.

- MGRO J1852+01: In the original Milagro map of the TeV sky, this source missed the statistical threshold for candidate sources. It has now been conclusively observed by HAWC and is a potential neutrino source considering its relatively large flux. Since the proper study of spectrum and extension of the source have not been performed by HAWC, we have studied the neutrino flux under different assumptions for the source's extension and spectrum. We find that IceCube should see this source in 5 years of data provided that the source is not extended. However, if the source is extended, 15 years is required to reach a significant level of observation.

- MGRO J2031+41: Due to the uncertainties associated with the origin of the flux of the Cygnus cocoon and $\gamma$-Cygni, a complete picture of this source is missing. Its extension and other $\mathrm{TeV}$ emissions in its vicinity have made it difficult for ACT experiments like VERITAS to measure the TeV flux from this source. Although previous studies indicated that observing the source would be challenging [20], using recent ARGO-YBJ and Fermi data, we argue that neutrino observations at the level of $3 \sigma$ may be possible in 10 years of IceCube data.

- MGRO J2019+37: We present an update on the neutrino observation from this source based on the spectrum measured by VERITAS, which has provided up to now the most precise measurement for the spectrum of the source up to $30 \mathrm{TeV}$. We show that IceCube is likely to observe the source in 15 years. This source is currently classified as a PWN. Thus, the detection of neutrinos from this region could point towards the production mechanism of neutrinos in a PWN as described in Refs. [26-28].

\section{Milagro sources}

After confirmation by HAWC [24, 25], the Milagro sources that we consider in this analysis are, as mentioned above, MGRO J1908+06, MGRO J1852+01, MGRO J2031+41, and MGRO J2019+37. In this section, we sum- 
marize the experimental information on these sources.

MGRO J1908+06: The source MGRO J1908+06 has been detected by large-acceptance air-shower detectors (EAS) like the Milagro experiment, see Refs. [23, 29, 30], and the ARGO-YBJ experiment [31]. This source has been detected also by ACTs, like HESS [32], which finds a spectrum with no evidence of a cut-off for energies $<20 \mathrm{TeV}$. The HESS detector reports a flux systematically lower than the Milagro and ARGO-YBJ data. With better angular resolution, it could be that HESS detects the flux from a point source that is not resolved by the Milagro and ARGO-YBJ observation. MGRO J1908+06 has also been recently detected by VERITAS [33], and the flux reported is of the same order as the one measured by HESS. Also, the value recently reported by HAWC points towards a similar normalization [24].

We report in Table 1 the extension for MGRO J1908+06 observed by HESS, VERITAS, and ARGO-YBJ, while in Table 2 we report the flux measured by HESS and VERITAS. In Fig. 1, we have compiled the spectra for MGRO J1908+06 from the different experiments.

Finally, note that Fermi-LAT observes the pulsar PSR J1907+0602 within the extension of the Milagro source MGRO J1908+06 [34]. On the other hand, the large size and hard spectrum in TeV photons of MGRO J1908+06 are not characterstic of a PWN and perhaps consistent with a SNR. SNRs are suspected to be the sources of the highest energy cosmic rays in the Galaxy [35], see also Ref. [4, 5].

MGRO J1852+01: In the original Milagro survey, its statistical significance fell just below the statistical threshold to be a candidate source. With its recent observation by HAWC [25] MGRO J1852+01 becomes a plausible neutrino source candidate. The primary study of the six Milagro sources in Ref. [8] suggested that this source, due to its large flux, could considerably increase the probability of detecting neutrinos in IceCube in five years. The flux from a $3 \times 3$ degree region around MGRO J1852+01 is given by $d N / d E=\left(5.7 \pm 1.5_{\text {stat }} \pm\right.$ $\left.1.9_{\text {sys }}\right) \times 10^{-14} \mathrm{TeV}^{-1} \mathrm{~cm}^{-2} \mathrm{~s}^{-1}$ at the median detected energy of $12 \mathrm{TeV}$, assuming a differential source spectrum of $E^{-2.6}[36]$. In Table 1 and Table 2, the information on this source is summarized, while in Fig. 3 we show the best-fit spectrum from the Milagro collaboration.

MGRO J2031+41: The flux from MGRO J2031+41 has been measured by Milagro [22, 29, 30]; the measurement cannot distinguish between a power law and a power law with cut-off. This is also the case for the ARGO-YBJ observations [37]. The two experiments have comparable angular resolution. The flux measured by ARGO-YBJ [37] for this source is compatible with the one reported by Milagro, which extends to energy below 1 TeV.

In general, ACT experiments report much smaller fluxes for this source. Indeed, measurements by MAGIC [38], HEGRA [39], and Whipple [40] can account for just a few percent of the Milagro flux. The source has been recently studied by the VERITAS collaboration, which has reported a flux comparable to the one reported by MAGIC. In the current picture [41], there are several sources contributing to the emission of MGRO J2031+41: the cocoon, the $\gamma$-Cygni SNR, VER J2019+407, and TeV J2032+4130. The latter has been detected by both VERITAS and MAGIC. In conclusion, a complete picture and understanding of this source is still not given. New data have been presented by the ARGO-YBJ detector in Ref. [42], where the authors suggest identifying ARGO J2031+4157 as the TeV-energy counterpart of the Cygnus cocoon. For this reason, they report the best fit not only considering the ARGO-YBJ data but also including in their fit the Fermi-LAT data from the Cygnus cocoon. This results in a harder spectral index with significant consequences for the neutrino prediction. Since leptonic processes could contribute to the cocoon emission at the energies detected by Fermi-LAT, we might expect the purely hadronic component of MGRO J2031+41 to lie somewhere between the two fits obtained by the ARGO-YBJ collaboration. We report in Table 1 the extension of MGRO J2031+41 as given by the ARGO-YBJ experiment, while in Table 2 we show the fluxes obtained with and without the inclusion of the Fermi-LAT data in the fit. In Fig. 5, we report the spectra for MGRO J2031+41 from different experiments. Note that we do not report the measurements by HEGRA [39] and Whipple [40], but these are in agreement with the MAGIC results.

MGRO J2019+37: The flux of the source MGRO J2019+37 has been measured by Milagro, see Refs. [22, 29, 30], reporting a power-law with energy cut-off as best fit. This source has not been detected by the ARGO-YBJ detector, which instead set $90 \%$ C.L. upper bounds on the flux [37]. Additionally, a limit on the flux at $115 \mathrm{TeV}$ 


\begin{tabular}{l||c|c|c}
\hline Source & Type & $\sigma_{\text {ext }}(\mathrm{ACT})$ & $\sigma_{\text {ext }}($ EAS $)$ \\
\hline \hline MGRO J1908+06 & UNID & & $0.49^{\circ} \pm 0.22^{\circ}[37]$ \\
$\hookrightarrow$ ARGO-YBJ & & $0.34^{\circ}{ }_{-0.03}^{+0.04}[32]$ & \\
$\hookrightarrow$ HESS J1908+063 & & $0.44^{\circ} \pm 0.02^{\circ}[33]$ & \\
$\hookrightarrow$ VERITAS & & & Milagro: $3^{\circ} \times 3^{\circ}$ search region [36] \\
\hline MGRO J1852+01 & UNID & & $1.8^{\circ} \pm 0.5^{\circ}[42]$ \\
\hline MGRO J2031+41 & UNID & & Milagro: $0.7^{\circ}[22]$ \\
$\hookrightarrow$ ARGO J2031+4157 & & & \\
\hline MGRO J2019+37 & PWN & $\sim 0.35^{\circ}[44]$ & \\
\hline VER J2019+368 & & & \\
\hline
\end{tabular}

Table 1: Extensions of the sources as reported by different experiments, air Cherenkov telescopes or extensive air-shower detectors. For the source MGRO J2031+41, we do not report the extension of the corresponding sources detected by ACT experiments, since the flux of these sources is much smaller than the one reported by the Milagro collaboration, see text for details. Note that the four sources have been recently detected by HAWC [24, 25].

\begin{tabular}{|c|c|}
\hline Source & $E_{\gamma}^{\text {norm }} ; \quad d N_{\gamma}^{12} / d E_{\gamma}$ at $E_{\gamma}^{\text {norm }} ; \quad \alpha_{\gamma}(\mathrm{ACT}$ or EAS) \\
\hline $\begin{array}{l}\text { MGRO J1908+06 } \\
\hookrightarrow \text { HESS J1908+063 } \\
\hookrightarrow \text { VERITAS }\end{array}$ & $\begin{array}{ccc}1 \mathrm{TeV} ; & 4.14 \pm 0.32_{\text {stat }} \pm 0.83_{\text {sys }} ; & 2.10 \pm 0.07_{\text {stat }} \pm 0.2_{\text {sys }}[32] \\
1 \mathrm{TeV} ; & 4.23 \pm 0.41_{\text {stat }} \pm 0.85_{\text {sys }} ; & 2.20 \pm 0.10_{\text {stat }} \pm 0.20_{\text {sys }}[33]\end{array}$ \\
\hline $\begin{array}{l}\text { MGRO J1852+01 } \\
\hookrightarrow \text { Milagro }\end{array}$ & $12 \mathrm{TeV} ; \quad\left(5.7 \pm 1.5_{\text {stat }} \pm 1.9_{\text {sys }}\right) \times 10^{-2} ; \quad 2.6[36]$ \\
\hline $\begin{array}{l}\text { MGRO J2031+41 } \\
\hookrightarrow \text { ARGO J2031+4157 }\end{array}$ & $\begin{aligned} & \text { w/o Fermi-LAT: } \\
& 1 \mathrm{TeV} ;(2.5 \pm 0.4) \times 10 ; \quad 2.6 \pm 0.3[42] \\
& \text { w Fermi-LAT: } \\
& 0.1 \mathrm{TeV} ;(3.5 \pm 0.3) \times 10^{3} ; \quad 2.16 \pm 0.04[42]\end{aligned}$ \\
\hline $\begin{array}{l}\text { MGRO J2019+37 } \\
\hookrightarrow \text { VER J2019+368 }\end{array}$ & $5 \mathrm{TeV} ; \quad\left(8.1 \pm 0.7_{\text {stat }} \pm 1.6_{\text {sys }}\right) \times 10^{-2} ; \quad 1.75 \pm 0.08_{\text {stat }} \pm 0.2_{\text {sys }}[44]$ \\
\hline
\end{tabular}

Table 2: Flux in units of $10^{-12} \mathrm{TeV}^{-1} \mathrm{~cm}^{-2} \mathrm{~s}^{-1}$ at a specific energy $E_{\gamma}^{\text {norm }}$ and spectral index $\alpha_{\gamma}$ as recently reported by air Cherenkov telescopes or extensive air-shower detectors experiments.

has been inferred through the CASA-MIA experiment [43].

The Milagro source MGRO J2019+37 has been recently detected by VERITAS. In Ref. [44], the collaboration reported two sources in the region of MGRO J2019+37: the faint point-like source VER J2016+371 and the bright extended source VER J2019+368. This second source is likely to account for the bulk of the Milagro emission. The VERITAS collaboration reported a very low spectral index for this source on the order of 1.75, between 1-30 TeV. We list in Table 1 the extension for MGRO J2019+37 as given by VERITAS and the Milagro 2012 release, and in Table 2 the value of the flux reported by the VERITAS experiment. In Fig. 6, we show the data for MGRO J2019+37 from different experiments.

\section{Gamma rays and neutrino flux}

The gamma-ray flux in the TeV energy range can be parametrized in terms of a spectral slope $\alpha_{\gamma}$, an energy $E_{c u t, \gamma}$ where the accelerator cuts off, and a normalization $k_{\gamma}$ :

$$
\frac{d N_{\gamma}\left(E_{\gamma}\right)}{d E_{\gamma}}=k_{\gamma}\left(\frac{E_{\gamma}}{\mathrm{TeV}}\right)^{-\alpha_{\gamma}} \exp \left(-\sqrt{\frac{E_{\gamma}}{E_{c u t, \gamma}}}\right) .
$$




\begin{tabular}{|l||c|c|}
\hline Source & $\sigma_{\text {eff }}($ point-like; extended $)$ & Flux \\
\hline \hline MGRO J1908+06 & $0.64^{\circ} ; 0.72^{\circ}$ & HESS [32] \\
MGRO J1852+01 & $0.64^{\circ} ; 1.63^{\circ}$ & Milagro [36] \\
MGRO J2031+41 & $-; 1.91^{\circ}$ & ARGO-YBJ (+Fermi-LAT) [42] \\
MGRO J2019+37 & $0.64^{\circ} ; 0.73^{\circ}$ & VERITAS [44] \\
\hline
\end{tabular}

Table 3: Angular opening and normalization for the flux considered in the analysis. The angle is defined as $\sigma_{\text {eff }} \equiv \sqrt{\sigma_{\text {ext }}^{2}+\sigma_{\text {IC }}^{2}}$, where $\sigma_{\text {ext }}$ is the extension of the source reported by the collaboration (see Table 1 ), while $\sigma_{\text {IC }} \equiv 1.6 \Delta \xi_{\text {IC }}$, with $\Delta \xi_{\text {IC }}=0.4^{\circ}$, is the IceCube angular resolution. For the source MGRO J1908+06, we will use the normalization given by HESS, compatible with the one reported by VERITAS; see Table 2 for the specific values.

The estimate in Eq. 1 indicates that fluxes as large as $d N_{\gamma} / d E_{\gamma} \sim 10^{-12}-10^{-14}\left(\mathrm{TeV}^{-1} \mathrm{~cm}^{-2} \mathrm{~s}^{-1}\right)$ can be expected at energies of $\mathcal{O}(10 \mathrm{TeV})$.

Using the parametrization of Eq. 2, the neutrino fluxes at Earth can be described by the following expression [45, 46]:

$$
\frac{d N_{\nu_{\mu}+\bar{\nu}_{\mu}}\left(E_{\nu}\right)}{d E_{\nu}}=k_{\nu}\left(\frac{E_{\nu}}{\mathrm{TeV}}\right)^{-\alpha_{\nu}} \exp \left(-\sqrt{\frac{E_{\nu}}{E_{c u t, \nu}}}\right)
$$

where

$$
\begin{aligned}
& k_{\nu}=\left(0.694-0.16 \alpha_{\gamma}\right) k_{\gamma}, \\
& \alpha_{\nu}=\alpha_{\gamma}, \\
& E_{c u t, \nu}=0.59 E_{c u t, \gamma} .
\end{aligned}
$$

The number of throughgoing muon neutrinos from a source at zenith angle $\theta_{Z}$ is given by Ref. [8]:

$$
N_{e v}=t \int_{E_{\nu}^{\mathrm{th}}} d E_{\nu} \frac{d N_{\nu}\left(E_{\nu}\right)}{d E_{\nu}} \times A_{\nu}^{\mathrm{eff}}\left(E_{\nu}, \theta_{Z}\right),
$$

where we have summed over neutrino and antineutrino contributions. We will use the IceCube neutrino effective area reported in Ref. [21].

\section{Results}

Based on the updated information from gamma-ray experiments described in the previous section, we revisit the prospects for observing neutrinos from these sources with IceCube, using the effective area for the 86-string detector configuration [21]. This study updates a previous study of three of the sources [20] using Milagro [22, 23] and ARGO-YJB (2012) measurements [37]. For related studies of the neutrino emission from Milagro sources, see also Refs. [8, 9, 43, 47-50].

The new information from gamma-ray experiments turns out to be important for a better parametrization of the flux of the gamma-ray sources. The uncertainties in the normalization and spectrum of the sources can result in important variations in the prediction of the neutrino fluxes. In this context, using updated data is important to make more reliable predictions and more appropriate interpretations of potential IceCube observations.

After calculating the neutrino flux, we compute the number of through-going muon neutrinos in IceCube. These have been produced inside or below the detector by neutrinos that have traversed the Earth. Any background of cosmic ray muons has thus been filtered out and only atmospheric neutrinos remain as a background for the northern hemisphere sources in a detector located at the South Pole. For each source, we fix the flux normalization to the best-fit values listed in Table 2. The expected number of muon neutrinos per energy bin are shown in Figs. 1, 3, 5 and 6 for the four sources considered in the analysis. For MGRO J1908+06, we have fixed $\alpha_{\gamma}=2$, consistent with the value reported by HESS, and we have varied the cut-off energy from $30 \mathrm{TeV}$ up to $800 \mathrm{TeV}$. For MGRO J1852+01, besides assuming $\alpha_{\gamma}=2$, we have also considered $\alpha_{\gamma}=2.6$ because this is the spectrum 
assumed by the Milagro collaboration. For MGRO J2031+41, we have considered the best-fit values for $\alpha_{\gamma}$ provided by the ARGO-YBJ collaboration, considering also the case in which the Fermi-LAT data have been added to the fit. Finally, for MGRO J2019+37 we have considered the case of $\alpha_{\gamma} \sim 1.75$, the best-fit value reported by the VERITAS collaboration.

To calculate the number of background atmospheric neutrino events, we have integrated the atmospheric flux [51] over an opening angle $\Omega=\pi \sigma_{\text {eff }}^{2}$ around the direction of the source, where the angle $\sigma_{\text {eff }}=\sqrt{\sigma_{\text {ext }}^{2}+\sigma_{\text {IC }}^{2}}$. The values of $\sigma_{\text {ext }}$, the physical extension of the source, are reported in Table 1 , while $\sigma_{\text {IC }} \equiv 1.6 \Delta \xi_{\text {IC }}$. The angular resolution of the IceCube detector $\Delta \xi_{\mathrm{IC}}$ is $0.4^{\circ}$ at the energies relevant for this analysis [52]. This angular radius correspond to a solid angle that contains roughly $72 \%$ of the signal events from the source; see also Ref. [53] for a discussion. The difference in the neutrino fluxes assuming an extended and point source is important as can be seen from Table 3, with the biggest difference between these two assumptions for MGRO J1852+01. $[54]$ :

We have subsequently estimated the statistical significance for observing the sources using the analytic expression

$$
p_{\text {value }}=\frac{1}{2}\left[1-\operatorname{erf}\left(\sqrt{q_{0}^{o b s} / 2}\right)\right] \text {, }
$$

where $q_{0}^{o b s}$ is defined as

$$
q_{0}^{o b s} \equiv-2 \ln \mathcal{L}_{b, D}=2\left(Y_{b}-N_{D}+N_{D} \ln \left(\frac{N_{D}}{Y_{b}}\right)\right) .
$$

Here, $Y_{b}$ is the theoretical expectation for the background hypothesis, while $N_{D}$ is the estimated signal generated as the median of events Poisson-distributed around the signal plus background. We have considered the total number of events (not binned in energy) to have a closer prediction to what is done in the IceCube point-source searches $[21]$.

In Fig. 2, we show the results for MGRO J1908+06. For this source, recent ACT data have reported a spectral index $\alpha_{\gamma}$ that is compatible with $\sim 2$. Despite the fact that the ACTs' normalization is smaller than the one previously reported by Milagro, the hard spectral index makes the source an interesting candidate for neutrino detection. For this reason, we also estimated how the p-value depends on the threshold energy that can be reached in a realistic analysis. We find that a $3 \sigma$ discovery is possible in six years, if an energy threshold of about 5 TeV can be reached in the analysis, and that the spectrum extends to $E_{\text {cut }, \gamma}$ of $800 \mathrm{TeV}$. For the more conservative case that $E_{\text {cut }, \gamma} \sim 300 \mathrm{TeV}$, as expected for galactic sources able to explain the cosmic-ray spectrum up to the knee, then an energy threshold of about $10 \mathrm{TeV}$ would be required. Obtaining a $3 \sigma$ discovery at a specific energy threshold will indicate a particular value of the cut-off energy $E_{\text {cut }, \gamma}$.

In Fig. 4, we show the statistical significance for MGRO J1852+01. For this source, due to the lack of data, not only is the spectral index poorly known but also the morphology of the source, whether extended or point-like, is uncertain. For the point-like hypothesis, a $3 \sigma$ discovery can be reached in six years, independently of the energy cut-off $E_{\text {cut }, \gamma}$ and spectral index $\alpha_{\gamma}$ of the source, while more than 10 years are required if the source is extended.

In Fig. 5, we show the p-value for MGRO J2031+41. As explained in detail in the previous section, the origin of the gamma-ray emission from this source is not understood. Using the best fit obtained by the ARGO-YBJ collaboration in conjunction with the Fermi-LAT data, we find that a $3 \sigma$ discovery is possible with 10 years of IceCube data. If this is indeed realized, the IceCube data not only would point towards a hadronic emission at TeV energies for MGRO J2031+41 but would help clarify the origin of the gamma-ray emission from the cocoon.

In Fig. 6, we show the statistical significance for MGRO J2019+37. A detection of neutrinos from this source would be extremely interesting since it might point towards the mechanism described in Refs. [26-28] for neutrino production in PWNs. For this source, we expect to obtain a $3 \sigma$ discovery in roughly 15 years. Future data from HAWC on the spectrum of this source are important to confirm the hard spectral index, on the order of $\alpha_{\gamma} \sim 1.75$, reported by the VERITAS collaboration.

The Milagro collaboration has presented results on the energy spectrum of these sources obtained by unfolding of the data [22]. It is obviously important for other experiments to confirm the presence of a low-energy cut-off that they consistently find in the analysis of every source. In this context, the constraints that IceCube can set in the plane $\left(\alpha_{\gamma}, E_{c u t, \gamma}\right)$ with future data are important and complementary. We have therefore estimated the constraints set on $\alpha_{\gamma}$ and $E_{\text {cut, } \gamma}$ in the absence of a signal after 15 years of exposure with the complete 86-string IceCube 
detector. We have integrated the number of events from $1 \mathrm{TeV}$ to $1 \mathrm{PeV}$ in neutrino energy $E_{\nu}$ and defined the confidence level, C.L., as in Refs. [54-57]:

$$
\text { C.L. }=\frac{P_{(s+b)}}{1-P_{b}} .
$$

with $P_{(s+b)}$ and $P_{b}$ the p-values for the signal plus background and background-only hypothesis of the data, respectively; see Ref. [20] for details.

The results for the expected C.L. are presented in Fig. 7 for the four sources considered in this paper and for a running time of $t=15$ years. We have fixed the normalization to the best fit reported in Table 2 , while we have varied the values of the spectral index $\alpha_{\gamma}$ and the cut-off energy $E_{c u t, \gamma}$. As shown in the figure, for MGRO J1908+06, IceCube is able to constrain a major part of the values for $\alpha_{\gamma}$ reported by the HESS detector. In particular, for a spectral index as hard as $\alpha_{\gamma} \sim 2$, values of $E_{\text {cut }, \gamma}$ greater than $100 \mathrm{TeV}$ could be excluded at $95 \%$ C.L. For MGRO $\mathrm{J} 1852+01$, IceCube will exclude all the parameter space with $E_{\text {cut }, \gamma}$ greater than $30 \mathrm{TeV}$ at $95 \%$ C.L. For the source MGRO J2031+41, the allowed region of $\alpha_{\gamma}$ obtained considering ARGO-YBJ plus Fermi-LAT data will be excluded at $99 \%$ C.L., independently of the value of $E_{\mathrm{cut}, \gamma}$. Finally, for MGRO J2019+37, considering the standard value of $E_{\text {cut }, \gamma}$ of $300 \mathrm{TeV}$, hard values of the spectral index with $\alpha_{\gamma}<2$ will be excluded at $95 \%$ C.L.

As mentioned above, the Milagro collaboration has reported a low-energy cut-off in the spectra of the sources MGRO J1908+06, MGRO J2031+41 and MGRO J2019+37 [22, 23]. In this case, the combinations of $\alpha_{\gamma}$ and $E_{c u t, \gamma}$ that could be excluded at 95-99 \% C.L. using future IceCube data are important because they can independently probe the presence of a low-energy cut-off.

\section{Conclusions}

The highest energy survey of the Galactic plane has been performed by Milagro. This survey has identified bright sources in the nearby Cygnus star-forming region and in the inner part the Galaxy. Initially, the sources showed the expected behavior of PeVatrons. PeVatrons are the sources of cosmic rays in the "knee" region of the cosmic-ray spectrum that are expected to be sources of pionic gamma rays whose spectrum extends to several hundreds of $\mathrm{TeV}$ without a cut-off. Gamma rays from the decay of neutral pions are inevitably accompanied by neutrinos with a flux that is calculable.

In this paper, we re-evaluated the probability of observing four promising Milagro sources in IceCube. We used the updated information from air-Cherenkov and air-shower array experiments to estimate the flux of neutrinos. The prospects for observing these sources in IceCube is highly entangled with discrepancies in the detailed fluxes and morphologies measured by different experiments. Moreover, the uncertainty of the nature of these sources makes it more difficult to understand the observed spectrum. Different spectra and morphology of the sources correspond to different production mechanisms.

It should be noted that the discrepancy between measurements may arise from the difference in angular resolution between air-shower arrays and air-Cherenkov telescopes as well as from the range of energies in which they operate. Future results from HAWC will help resolve these discrepancies and reveal more information about the sources.

If the gamma rays are hadronic in origin, observation of an accompanying neutrino flux is likely over the lifetime of the IceCube experiment. Evidence from IceCube of neutrinos associated with these sources will greatly help in unraveling the nature of the sources. 

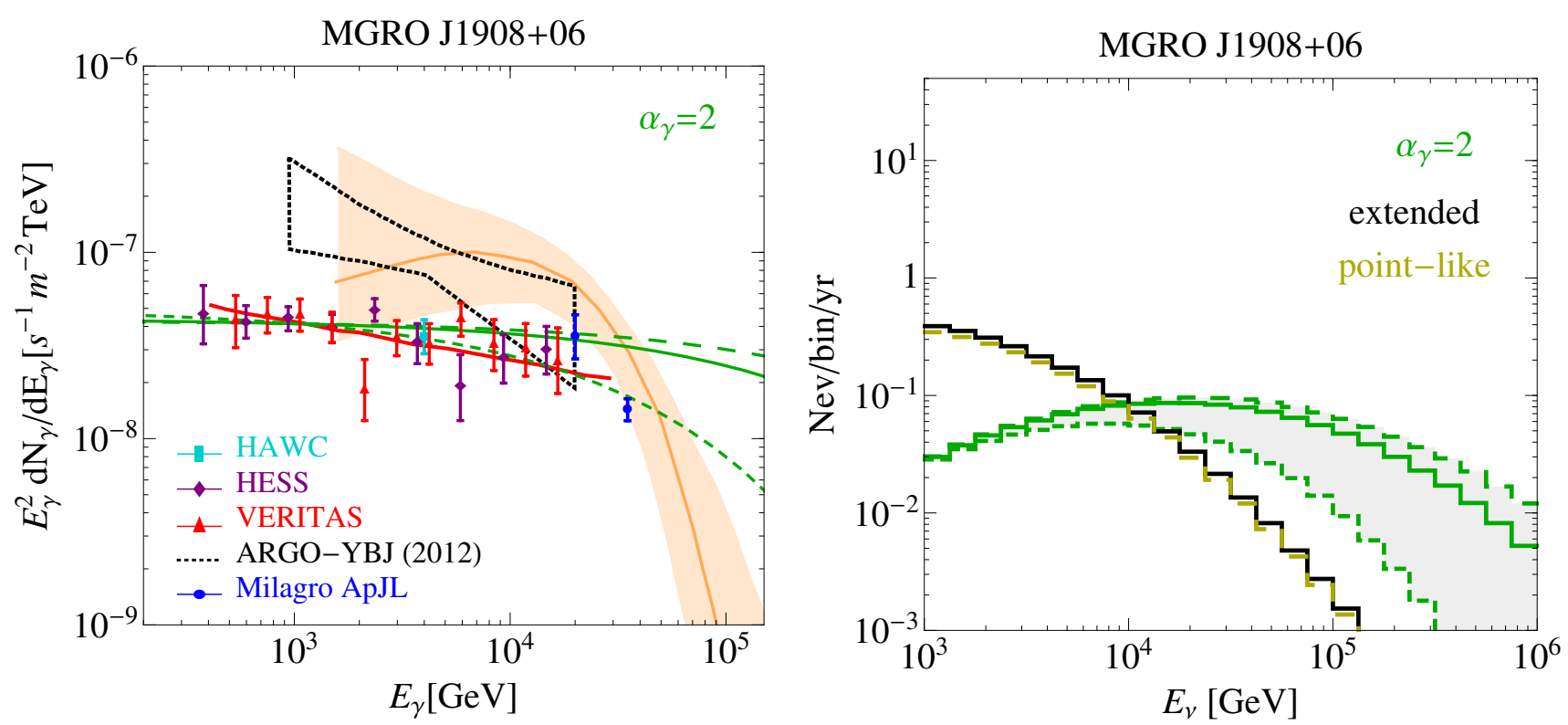

Figure 1: Left panel: We show in purple the data by HESS [32], in red the one from VERITAS [44], and in cyan the one from HAWC [24]. In blue we show the previous flux measurements by Milagro $[29,30]$, while the solid orange line and the shaded orange area show the best fit and the $1 \sigma$ band as reported in Ref. [23] by Milagro. The dotted area is the ARGO-YBJ $1 \sigma$ band [31]. With green lines we show the spectra obtained considering $\alpha_{\gamma}=2$ and fixing the normalization to the best fit reported in Table 2, where we also allowed the cut-off energy to vary: $E_{\text {cut }, \gamma}=30,300$, and $800 \mathrm{TeV}$ (short-dashed, solid, and long-dashed lines, in green). Right panel: We show the corresponding number of events for these spectra. The gray band encodes the uncertainty on the cut-off energy. With the black (gold dashed) line, we show the background from atmospheric neutrinos for extended (point-like) sources.
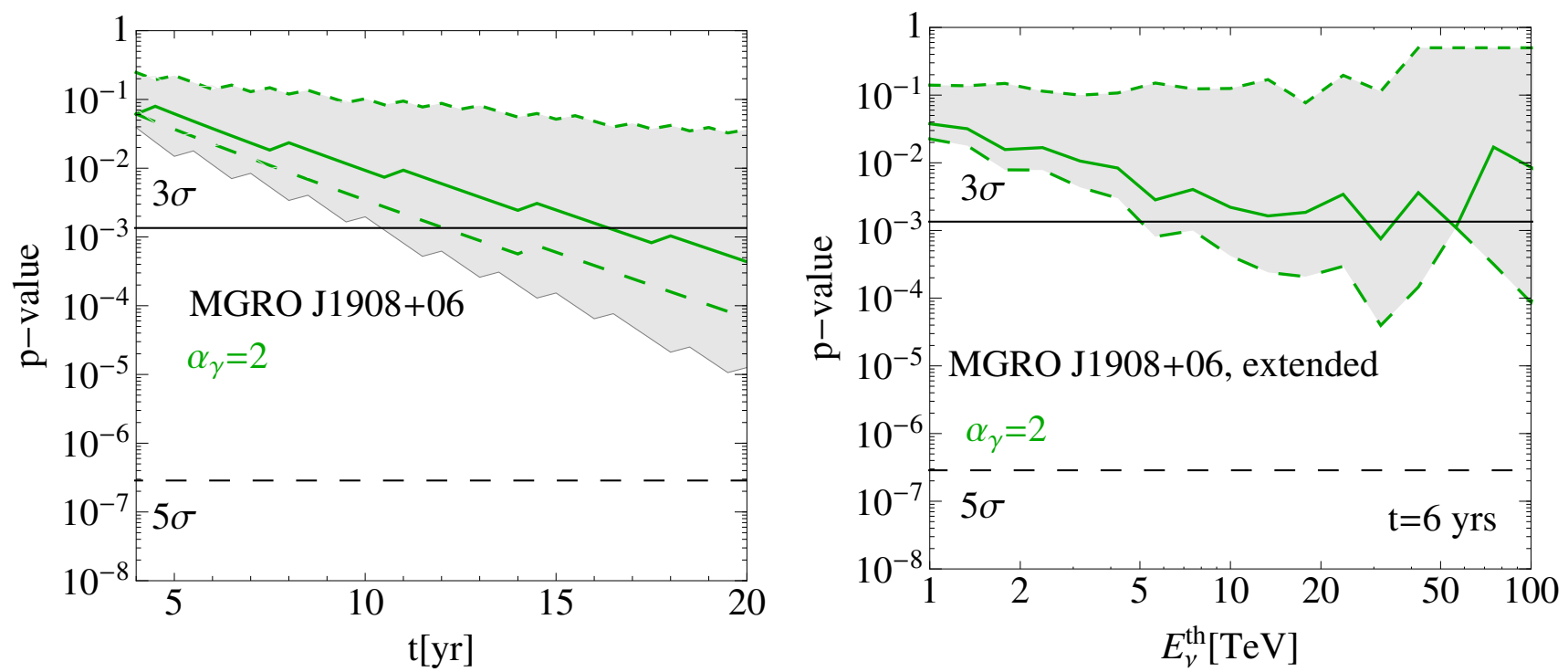

Figure 2: Left panel: p-values as a function of time, from 4 years to 20 years. The spectra have been fixed, as shown in Fig. 1. The gray band encodes the uncertainty due to different values of $E_{c u t, \gamma}$, and morphology, see Table. 3 . For the green lines we have considered the case of extended source. Right panel: Dependence of the p-value on the energy threshold $E_{\nu}^{t h}$. 

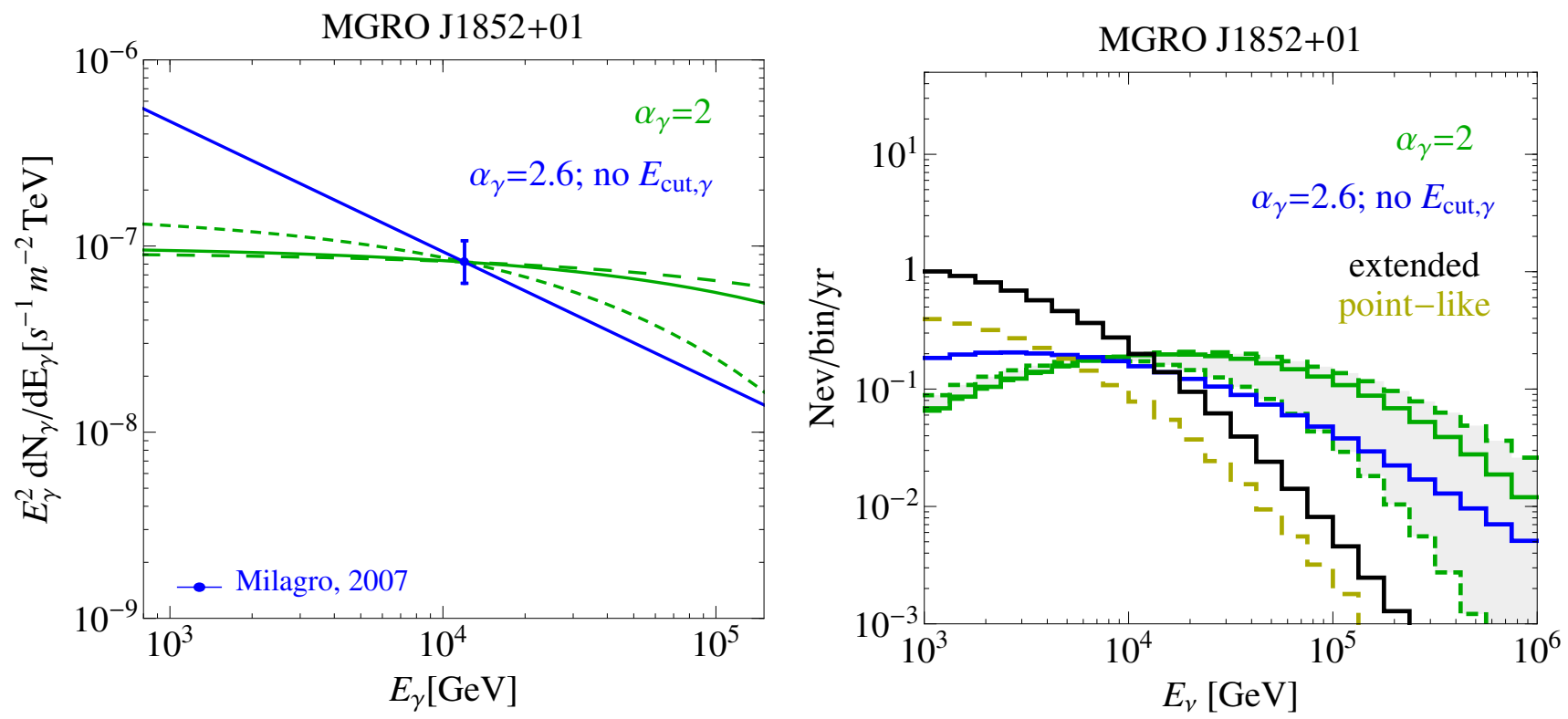

Figure 3: Left panel: We show in blue the value on the flux reported by the Milagro collaboration [36], which assumed an $E^{-2.6}$ spectrum. With green lines we show the spectra obtained considering $\alpha_{\gamma}=2$ and fixing the normalization to the best fit reported in Table 2, where we also allowed the cut-off energy to vary: $E_{\mathrm{cut}, \gamma}=30,300$, and $800 \mathrm{TeV}$ (short-dashed, solid, and long-dashed lines, in green). Right panel: Number of events for the spectra reported with green and blue lines in the left panel. The gray band encodes the uncertainty on the cut-off energy. With the black (gold dashed) line, we show the background from atmospheric neutrinos for extended (point-like) sources.
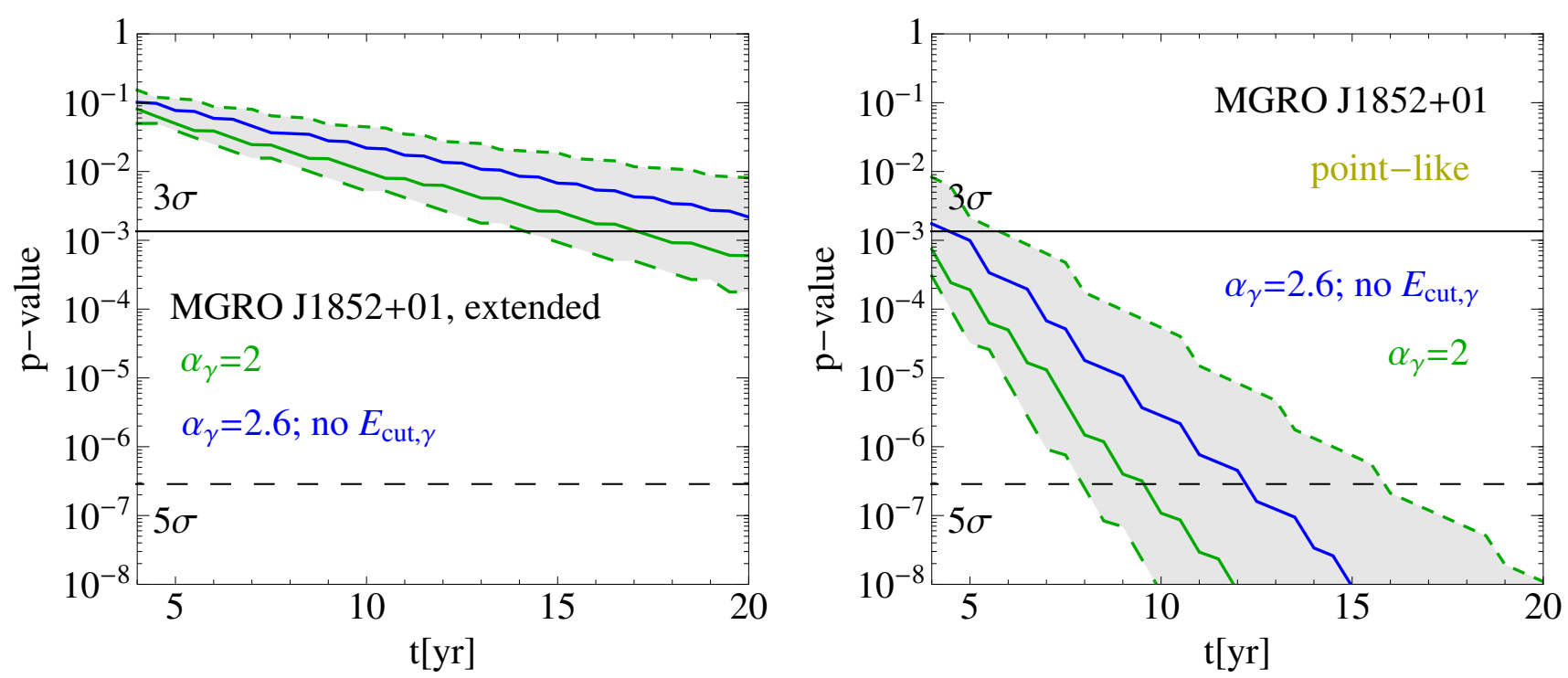

Figure 4: Left panel: p-values as a function of time, from 4 years to 20 years. The spectra have been fixed, as shown in Fig. 3 . The gray band encodes the uncertainty due to different values of $E_{c u t, \gamma}$. We assume the source to be extended. Right panel: We assume the source to be point-like. 

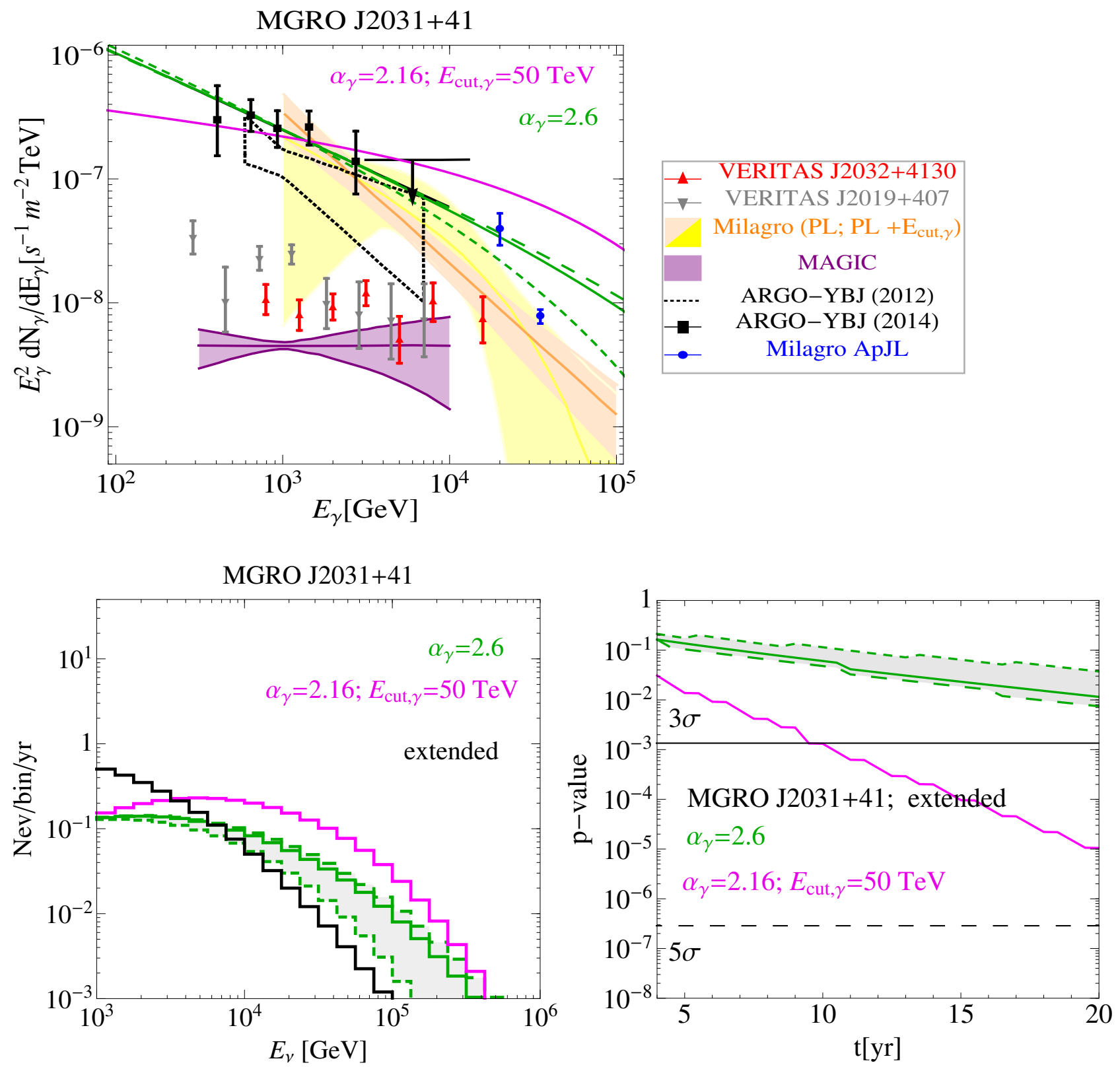

Figure 5: Upper panel: The black points show the data reported by ARGO-YBJ in Ref. [42], while the dotted region is the one reported in Ref. [37]. The previous flux measurements by Milagro are shown in blue [29, 30], while the orange/yellow area denotes the the powerlaw model/the power-law model with cut-off as reported in Ref. [22] by Milagro. With the purple band we report the measurements by MAGIC [38]. We report in red and grey the results from the VERITAS detector [41]. With green/magenta lines we show the spectra obtained fixing the parameters to the best fit reported in Table 2 for the case without/with Fermi data. In the case without Fermi data, we also allowed the cut-off energy to vary: $E_{\text {cut, } \gamma}=30,300$, and $800 \mathrm{TeV}$ (short-dashed, solid, and long-dashed lines, in green. Lower panel, left: Number of events for the spectra reported with green and magenta lines in the upper panel. The gray band encodes the uncertainty on the cut-off energy. With black lines, we show the background from atmospheric neutrinos. Lower panel, right: p-values as a function of time, from 4 years to 20 years. 

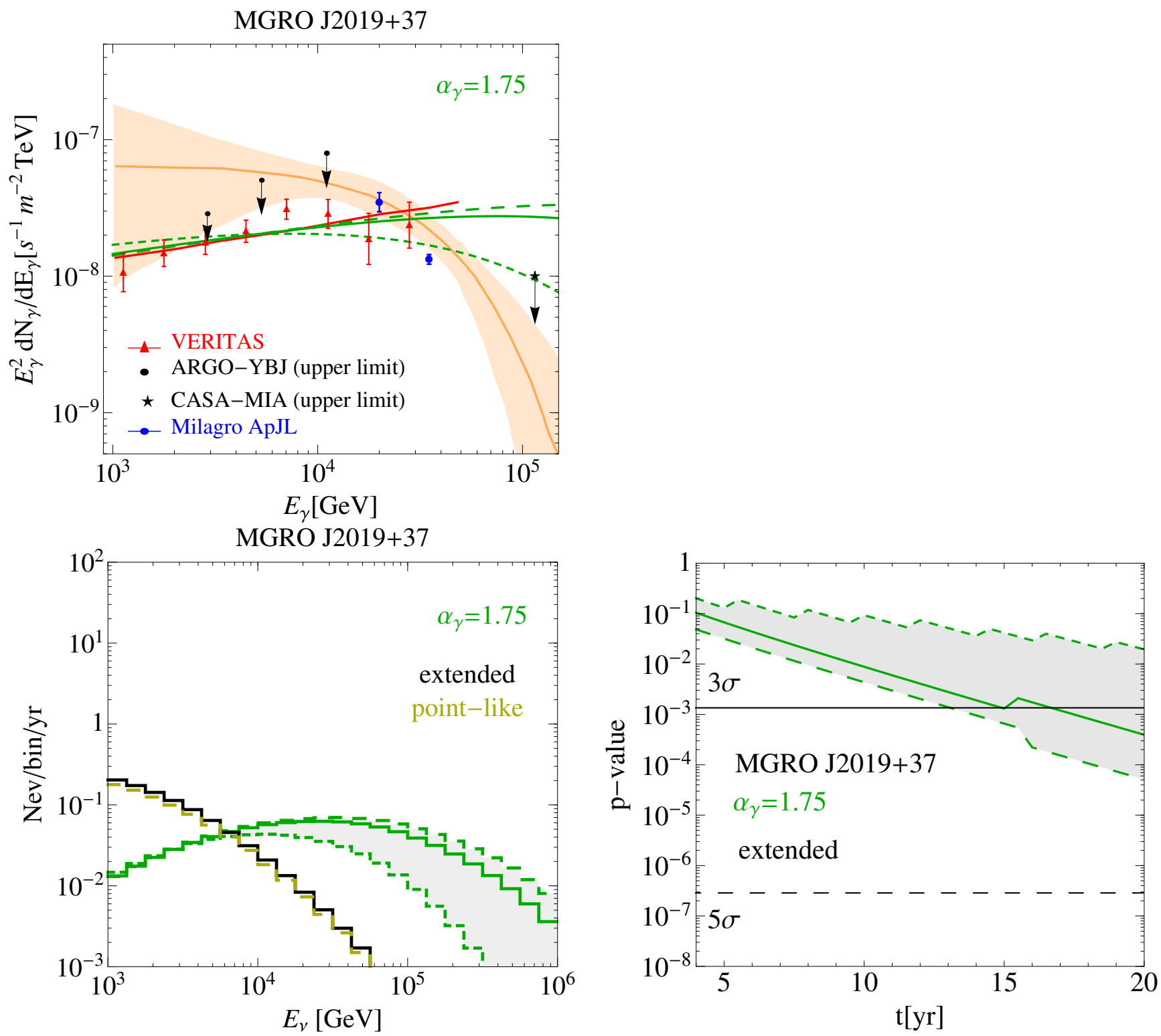

Figure 6: Upper panel: With red points, we report the VERITAS data [33]. With blue lines, we report the previous flux measurements by Milagro [29,30], while the continuous orange line and the shaded orange area represent the best fit and $1 \sigma$ band [22] as reported by Milagro. The $90 \%$ C.L. upper limits from ARGO-YBJ are shown in black [37], and the inferred CASA-MIA bound [43] is shown with a black star. With green lines we show the spectra obtained fixing the parameters to the best fit reported in Table 2 , where we also allowed the cut-off energy to vary: $E_{\text {cut }, \gamma}=30,300$, and $800 \mathrm{TeV}$ (short-dashed, solid, and long-dashed lines, in green). Lower panel, left: Number of events for the spectra reported with green and magenta lines in the left panel. The gray band encodes the uncertainty on the cut-off energy. With the black (gold dashed) line, we show the background from atmospheric neutrinos for extended (point-like) sources. Lower panel, right: p-values as a function of time, from 4 years to 20 years. 

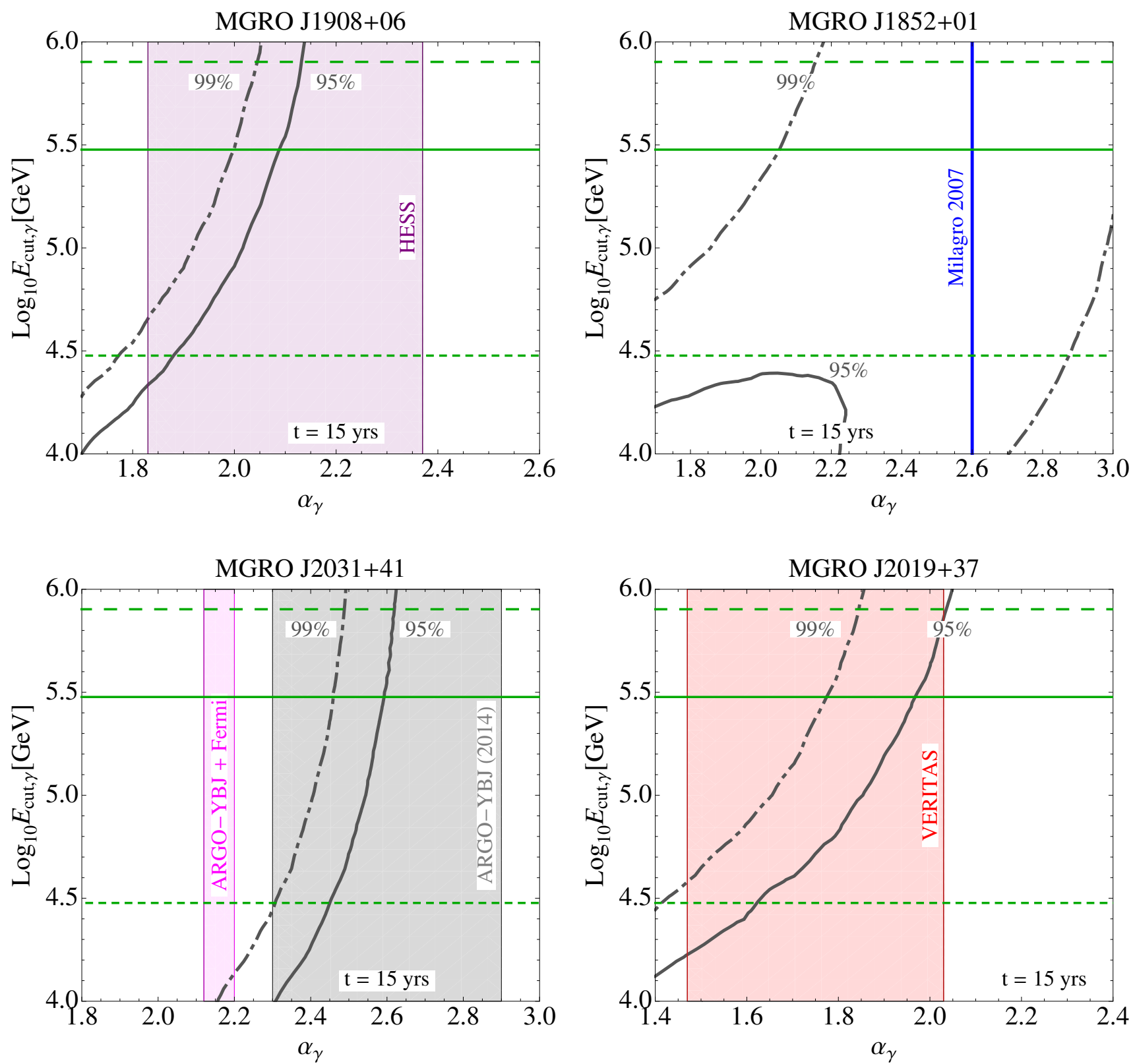

Figure 7: Upper panel: Values of $\alpha_{\gamma}$ and $E_{c u t, \gamma}$ excluded at 95\% (solid) and 99\% C.L. (dot-dashed) with 15 years of IceCube running with its 86-string configuration. The normalization has been fixed to the best fit reported by HESS [32] (left) and Milagro 2007 (right). We have assumed extended sources. With horizontal lines we denote the values $E_{\text {cut, },}=30$, 300, and $800 \mathrm{TeV}$ (short-dashed, solid, and long-dashed lines, in green). The purple region (left) denotes the values of $\alpha_{\gamma}$ reported by HESS. The blue line (right) denotes the value of $\alpha_{\gamma}$ considered by Milagro. Lower panel: The normalization has been fixed to the best fit reported by ARGO-YBJ without Fermi-LAT [37] (left) and VERITAS [44] (right). We have assumed extended sources. The gray/magenta region (left) denotes the values of $\alpha_{\gamma}$ reported by ARGO-YBJ without/with Fermi data. The red region (right) denotes the values of $\alpha_{\gamma}$ reported by VERITAS. 


\section{Acknowledgments}

We would like to thank Markus Ahlers, Chad Finley, M.C. Gonzalez-Garcia, Zigfried Hampel, Teresa Montaruli, Amanda Weinstein, and Tom Weisgarber for useful discussions. We would like to specially thank Jay Gallagher, Stefan Westerhoff, and Tova Yoast-Hull for their informative comments and discussions during this study. FH and AK research was supported in part by the U.S. National Science Foundation under Grants No. ANT-0937462 and PHY- 1306958 and by the University of Wisconsin Research Committee with funds granted by the Wisconsin Alumni Research Foundation. VN acknowledges hospitality at WIPAC where part of this work has been carried out. VN acknowledges support by Spanish MINECO through project FPA2012-31880, by Spanish MINECO (Centro de excelencia Severo Ochoa Program) under grant SEV-2012-0249. VN acknowledges financial support by the European Union through the ITN ELUSIVES H2020-MSCA-ITN-2015//674896 and the RISE INVISIBLESPLUS H2020-MSCA-RISE-2015//690575.

\section{References}

[1] M. Gonzalez-Garcia, F. Halzen, S. Mohapatra, Identifying Galactic PeVatrons with Neutrinos, Astropart.Phys. 31 (2009) $437-444$. arXiv:0902.1176, doi:10.1016/j.astropartphys.2009.05.002.

[2] M. Ahlers, P. Mertsch, S. Sarkar, On cosmic ray acceleration in supernova remnants and the FERMI/PAMELA data, Phys. Rev. D80 (2009) 123017. arXiv:0909.4060, doi:10.1103/PhysRevD.80.123017.

[3] A. A. Abdo, et al., Discovery of TeV Gamma-Ray Emission from the Cygnus Region of the Galaxy, Astrophys. J. 658 (2007) L33-L36. arXiv:astro-ph/0611691, doi:10.1086/513696.

[4] S. Gabici, F. A. Aharonian, Searching for galactic cosmic ray pevatrons with multi-TeV gamma rays and neutrinos, Astrophys.J. 665 (2007) L131. arXiv:0705.3011, doi:10.1086/521047.

[5] M. Ackermann, et al., Detection of the Characteristic Pion-Decay Signature in Supernova Remnants, Science 339 (2013) 807. arXiv:1302.3307, doi:10.1126/science.1231160.

[6] A. A. Abdo, Observations of the young supernova remnant RX J1713.7-3946 with the Fermi Large Area Telescope, Astrophys. J. 734 (2011) 28. arXiv:1103.5727, doi:10.1088/0004-637X/734/1/28.

[7] A. Abramowski, et al., Acceleration of petaelectronvolt protons in the Galactic Centre, Nature 531 (2016) 476. arXiv:1603.07730, doi:10.1038/nature17147.

[8] F. Halzen, A. Kappes, A. O'Murchadha, Prospects for identifying the sources of the Galactic cosmic rays with IceCube, Phys. Rev. D78 (2008) 063004. arXiv:0803.0314, doi:10.1103/PhysRevD.78.063004.

[9] A. Kappes, F. Halzen, A. O. Murchadha, Prospects of identifying the sources of the galactic cosmic rays with IceCube, Nucl.Instrum.Meth. A602 (2009) 117-119. doi:10.1016/j.nima.2008.12.049.

[10] F. Halzen, High-energy neutrino astrophysics, Nat Phys advance online publication. doi:10.1038/nphys3816.

[11] A. M. Taylor, S. Gabici, F. Aharonian, Galactic halo origin of the neutrinos detected by IceCube, Phys.Rev. D89 (10) (2014) 103003. arXiv:1403.3206, doi:10.1103/PhysRevD.89.103003.

[12] D. Gaggero, D. Grasso, A. Marinelli, A. Urbano, M. Valli, The gamma-ray and neutrino sky: A consistent picture of Fermi-LAT, Milagro, and IceCube results, Astrophys. J. 815 (2) (2015) L25. arXiv:1504.00227, doi:10.1088/2041-8205/815/2/L25.

[13] M. Ahlers, Y. Bai, V. Barger, R. Lu, Galactic neutrinos in the TeV to PeV range, Phys. Rev. D93 (1) (2016) 013009. arXiv:1505.03156, doi:10.1103/PhysRevD.93.013009.

[14] A. Palladino, F. Vissani, Extragalactic plus Galactic model for IceCube neutrino events, Astrophys. J. 826 (2) (2016) 185. arXiv:1601.06678, doi:10.3847/0004-637X/826/2/185.

[15] D. Fox, K. Kashiyama, P. Mészarós, Sub-PeV Neutrinos from TeV Unidentified Sources in the Galaxy, Astrophys.J. 774 (2013) 74. arXiv:1305.6606, doi:10.1088/0004-637X/774/1/74.

[16] C. Lunardini, S. Razzaque, High Energy Neutrinos from the Fermi Bubbles, Phys.Rev.Lett. 108 (2012) 221102. arXiv:1112.4799, doi:10.1103/PhysRevLett.108.221102.

[17] C. Lunardini, S. Razzaque, K. T. Theodoseau, L. Yang, Neutrino Events at IceCube and the Fermi Bubbles, Phys.Rev. D90 (2) (2014) 023016. arXiv:1311.7188, doi:10.1103/PhysRevD.90.023016.

[18] Y. Bai, A. J. Barger, V. Barger, R. Lu, A. D. Peterson, J. Salvado, Neutrino Lighthouse at Sagittarius A*, Phys. Rev. D90 (6) (2014) 063012. arXiv:1407.2243, doi:10.1103/PhysRevD.90.063012.

[19] S. Adrian-Martinez, et al., Searches for Point-like and extended neutrino sources close to the Galactic Centre using the ANTARES neutrino Telescope, Astrophys.J. 786 (2014) L5. arXiv:1402.6182, doi:10.1088/2041-8205/786/1/L5.

[20] M. Gonzalez-Garcia, F. Halzen, V. Niro, Reevaluation of the Prospect of Observing Neutrinos from Galactic Sources in the Light of Recent Results in Gamma Ray and Neutrino Astronomy, Astropart.Phys. 57-58 (2014) 39-48. arXiv:1310.7194, doi:10.1016/j.astropartphys.2014.04.001.

[21] M. Aartsen, et al., Searches for Extended and Point-like Neutrino Sources with Four Years of IceCube Data arXiv:1406.6757, doi:10.1088/0004-637X/796/2/109.

[22] A. Abdo, U. Abeysekara, B. Allen, T. Aune, D. Berley, et al., Spectrum and Morphology of the Two Brightest Milagro Sources in the Cygnus Region: MGRO J2019+37 and MGRO J2031+41, Astrophys.J. 753 (2012) 159. arXiv:1202.0846, doi:10.1088/0004$637 \mathrm{X} / 753 / 2 / 159$.

[23] A. J. Smith, A Survey of Fermi Catalog Sources using Data from the Milagro Gamma-Ray Observatory arXiv:1001.3695. 
[24] A. U. Abeysekara, et al., Search for TeV Gamma-Ray Emission from Point-like Sources in the Inner Galactic Plane with a Partial Configuration of the HAWC Observatory, Astrophys. J. 817 (1) (2016) 3. arXiv:1509.05401, doi:10.3847/0004-637X/817/1/3.

[25] A. Sandoval, Highlights from hawc, 6th international symposium on high-energy gamma-ray astronomy (gamma2016), heidelberg, germany, july $11-15,2016$.

[26] M. Lemoine, K. Kotera, J. Pétri, On ultra-high energy cosmic ray acceleration at the termination shock of young pulsar winds, JCAP 1507 (2015) 016. arXiv:1409.0159, doi:10.1088/1475-7516/2015/07/016.

[27] E. Amato, D. Guetta, P. Blasi, Signatures of high energy protons in pulsar winds, Astron. Astrophys. 402 (2003) $827-836$. arXiv:astro-ph/0302121, doi:10.1051/0004-6361:20030279.

[28] I. Di Palma, D. Guetta, E. Amato, Revised predictions of neutrino fluxes from Pulsar Wind NebulaearXiv:1605.01205.

[29] A. Abdo, B. T. Allen, D. Berley, S. Casanova, C. Chen, et al., TeV Gamma-Ray Sources from a Survey of the Galactic Plane with Milagro, Astrophys.J. 664 (2007) L91-L94. arXiv:0705.0707, doi:10.1086/520717.

[30] A. Abdo, B. Allen, T. Aune, D. Berley, C. Chen, et al., Milagro Observations of TeV Emission from Galactic Sources in the Fermi Bright Source List, Astrophys.J. 700 (2009) L127-L131. arXiv:0904.1018, doi:10.1088/0004-637X/700/2/L127, 10.1088/0004$637 \mathrm{X} / 703 / 2 / \mathrm{L} 185$.

[31] Observation of the TeV gamma-ray source MGRO J1908+06 with ARGO-YBJ, Astrophys.J. 760 (2012) 110. arXiv:1207.6280, doi:10.1088/0004-637X/760/2/110.

[32] F. Aharonian, Detection of Very High Energy radiation from HESS J1908+063 confirms the Milagro unidentified source MGRO J1908+06, Astron.Astrophys. 499 (2009) 723. arXiv:0904.3409, doi:10.1051/0004-6361/200811357.

[33] E. Aliu, S. Archambault, T. Aune, B. Behera, M. Beilicke, et al., Investigating the TeV Morphology of MGRO J1908+06 with VERITAS, Astrophys.J. 787 (2014) 166. arXiv:1404.7185, doi:10.1088/0004-637X/787/2/166.

[34] A. Abdo, A. Abdo, PSR J1907+0602: A Radio-Faint Gamma-Ray Pulsar Powering a Bright TeV Pulsar Wind Nebula, Astrophys.J. 711 (2010) 64-74. arXiv:1001.0792, doi:10.1088/0004-637X/711/1/64.

[35] W. Baade, F. Zwicky, On super-novae, Proceedings of the National Academy of Sciences 20 (5) (1934) $254-259$. doi:10.1073/pnas.20.5.254.

[36] A. A. Abdo, Discovery of localized tev gamma-ray sources and diffuse tev gamma-ray emission from the galactic plane with milagro using a new background rejection technique, Ph.D. thesis, Michigan State University, Department of Physics and Astronomy (2007).

[37] B. Bartoli, P. Bernardini, X. Bi, C. Bleve, I. Bolognino, et al., Observation of TeV gamma rays from the Cygnus region with the ARGO-YBJ experiment, Astrophys.J. 745 (2012) L22. arXiv:1201.1973, doi:10.1088/2041-8205/745/2/L22.

[38] J. Albert, et al., MAGIC observations of the unidentified TeV gamma-ray source TeV J2032+4130, Astrophys.J. 675 (2008) L25-L28. arXiv:0801.2391, doi:10.1086/529520.

[39] F. Aharonian, et al., The Unidentified TeV source (TeV J2032+4130) and surrounding field: Final HEGRA IACT-system results, Astron.Astrophys. 431 (2005) 197-202. arXiv:astro-ph/0501667.

[40] M. J. Lang, D. Carter-Lewis, D. Fegan, S. Fegan, A. Hillas, et al., Evidence for TeV gamma ray emission from TeV j2032+4130 in whipple archival data, Astron.Astrophys. 423 (2004) 415-419. arXiv:astro-ph/0405513, doi:10.1051/0004-6361:20041021.

[41] A. Weinstein, Pulsar Wind Nebulae and Cosmic Rays: A Bedtime Story, Nucl.Phys.Proc.Suppl. 256-257 (2014) 136-148. arXiv:1411.2532, doi:10.1016/j.nuclphysbps.2014.10.017.

[42] B. Bartoli, et al., Identification of the TeV Gamma-ray Source ARGO J2031+4157 with the Cygnus Cocoon, Astrophys. J. 790 (2) (2014) 152. arXiv:1406.6436, doi:10.1088/0004-637X/790/2/152.

[43] J. F. Beacom, M. D. Kistler, Dissecting the Cygnus Region with TeV Gamma Rays and Neutrinos, Phys.Rev. D75 (2007) 083001. arXiv:astro-ph/0701751, doi:10.1103/PhysRevD.75.083001.

[44] E. Aliu, T. Aune, B. Behera, M. Beilicke, W. Benbow, et al., Spatially resolving the very high energy emission from MGRO J2019+37 with VERITAS, Astrophys.J. 788 (2014) 78. arXiv:1404.1841, doi:10.1088/0004-637X/788/1/78.

[45] S. Kelner, F. A. Aharonian, V. Bugayov, Energy spectra of gamma-rays, electrons and neutrinos produced at proton-proton interactions in the very high energy regime, Phys.Rev. D74 (2006) 034018. arXiv:astro-ph/0606058, doi:10.1103/PhysRevD.74.034018, 10.1103/PhysRevD.79.039901.

[46] A. Kappes, J. Hinton, C. Stegmann, F. A. Aharonian, Potential Neutrino Signals from Galactic Gamma-Ray Sources, Astrophys.J. 656 (2007) 870-896. arXiv:astro-ph/0607286, doi:10.1086/508936, 10.1086/518161.

[47] F. Halzen, A. O Murchadha, Neutrinos from Cosmic Ray Accelerators in the Cygnus Region of the Galaxy, Phys.Rev. D76 (2007) 123003. arXiv:0705.1723, doi:10.1103/PhysRevD.76.123003.

[48] F. Vissani, F. Aharonian, Galactic Sources of High-Energy Neutrinos: Highlights, Nucl.Instrum.Meth. A692 (2012) 5-12. arXiv:1112.3911, doi:10.1016/j.nima.2011.12.079.

[49] F. Vissani, F. Aharonian, N. Sahakyan, On the Detectability of High-Energy Galactic Neutrino Sources, Astropart.Phys. 34 (2011) 778-783. arXiv:1101.4842, doi:10.1016/j.astropartphys.2011.01.011.

[50] C. Tchernin, J. Aguilar, A. Neronov, T. Montaruli, Neutrino signal from extended Galactic sources in IceCube, Astron.Astrophys. 560 (2013) A67. arXiv:1305.4113, doi:10.1051/0004-6361/201321801.

[51] M. Honda, T. Kajita, K. Kasahara, S. Midorikawa, Improvement of low energy atmospheric neutrino flux calculation using the JAM nuclear interaction model, Phys.Rev. D83 (2011) 123001. arXiv:1102.2688, doi:10.1103/PhysRevD.83.123001.

[52] M. G. Aartsen, et al., Evidence for Astrophysical Muon Neutrinos from the Northern Sky with IceCube, Phys. Rev. Lett. 115 (8) (2015) 081102. arXiv:1507.04005, doi:10.1103/PhysRevLett.115.081102.

[53] D. Alexandreas, D. Berley, S. Biller, G. Dion, J. Goodman, et al., Point source search techniques in ultrahigh-energy gamma-ray astronomy, Nucl.Instrum.Meth. A328 (1993) 570-577. doi:10.1016/0168-9002(93)90677-A.

[54] Procedure for the LHC Higgs boson search combination in summer 2011.

[55] T. Junk, Confidence level computation for combining searches with small statistics, Nucl.Instrum.Meth. A434 (1999) $435-443$. arXiv:hep-ex/9902006, doi:10.1016/S0168-9002(99)00498-2.

[56] A. L. Read, Modified frequentist analysis of search results (The CL(s) method). 
[57] J. Beringer, et al., Review of Particle Physics (RPP), Phys.Rev. D86 (2012) 010001. doi:10.1103/PhysRevD.86.010001. 